\title{
Current status and prospects of muonium spectroscopy at PSI
}

\author{
Ben Ohayon, Zakary Burkley and Paolo Crivelli ${ }^{\star}$
}

ETH Zurich, Institute of Particle Physics and Astrophysics, 8093 Zurich, Switzerland

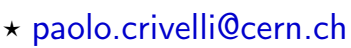

PAUL SCHERRER INSTITUT

Review of Particle Physics at PSI

doi:10.21468/SciPostPhysProc.5

\begin{abstract}
Recent and ongoing developments of low energy muon beamlines are heralding a new era of precision Muonium spectroscopy. While past spectroscopic measurements of Muonium were performed at pulsed muon facilities and were statistically limited, the advent of continuous low energy muon beams, such as at the LEM beamline at PSI, paired with the development of efficient muon-muonium converters and laser advancements, will overcome these limitations. Current experiments presently underway at the LEM facility and in the near future at the muCool beamline, which is under development at PSI, aim to improve the precision of both the 1S-2S transition determination and Lamb shift by several orders of magnitude. In this Chapter we give an overview of the current status and future prospects of these activities at PSI, highlighting how their projected significance fits into a broader context of other ongoing efforts worldwide.
\end{abstract}

(c) (1) Copyright B. Ohayon et al.

This work is licensed under the Creative Commons

Attribution 4.0 International License.

Published by the SciPost Foundation.
Received 09-06-2021

Accepted 29-06-2021

Published 06-09-2021

doi:10.21468/SciPostPhysProc.5.029

\subsection{Introduction}

The usefulness of precision spectroscopy for atomic systems with a hadronic nucleus is limited by our knowledge of quantum chromodynamics (QCD), which is not yet tractable at low energies. As an example, the hyperfine structure of the ground state of hydrogen was measured to better than the part-per-trillion (ppt) precision half a century ago but theoretical calculations are limited by proton structure and other hadronic effects to the level of partsper-million (ppm) [1]. Pure leptonic systems, such as positronium (Ps) and Muonium (M), are hydrogenic atoms composed of point-like particles. As such, they are devoid of finite-size effects and largely free of other hadronic contributions, making them ideal for determining fundamental constants, testing bound-state QED, and searching for new physics. Specific scenarios include the search for dark-sector particles and new muonic forces [2], as well as testing Lorentz and CPT symmetry [3].

Ps spectroscopy is an active field with current efforts focused on measuring optical and microwave transitions from its ground [4] and first-excited states $[5,6]$. The recent measurement of the Ps $n=2$ fine-structure is 4.5 standard deviations from its calculated value [6], 
which motivates further investigations of this system. The linewidth of low-lying transitions in Ps is inherently limited by the triplet annihilation lifetime of $142 \mathrm{~ns}$ in the ground-state and $1136 \mathrm{~ns}$ in the $2 \mathrm{~S}$ state. Its light mass, and corresponding high velocity, poses great challenges to determining first and second-order Doppler effects.

With a longer lifetime of 2196.9803(22) ns [7], limited by the muon decay, and a larger mass, $\mathrm{M}$ is a more suitable candidate for precision spectroscopy experiments. Past M spectroscopy experiments were conducted between 1980-2000 at TRIUMF, RAL and LAMPF (see [8] for a recent review). As a result of the difficulty in obtaining a high flux of $\mu^{+}$, and the necessity to slow down the muons so that $\mathrm{M}$ can be formed efficiently, all past $\mathrm{M}$ spectroscopy experiments were essentially limited by statistics, or statistics-related systematic effects [8]. With its intense $\mu^{+}$beam, PSI harbours tremendous opportunities for improving M spectroscopy experiments. Higher statistics makes it possible to implement experimental techniques which are systematically more robust and precise. In this respect the Low-Energy-Muon (LEM) beamline at PSI plays a crucial role.

The development of the LEM beamline was motivated by the desire to apply the Muon Spin Rotation ( $\mu \mathrm{SR})$ technique to surface and thin film physics [9]. A high intensity surface $(E=4.1 \mathrm{MeV}$ ) muon beam from the $\mu \mathrm{E} 4$ beamline [10] is moderated to $\sim 15 \mathrm{eV}$ by injecting it into a solid noble gas layer [9]. The beam is then re-accelerated to energies tunable in the range $1-30 \mathrm{keV}$. The availability of an intense $10^{4} / \mathrm{s} \mu^{+}$beam in this energy range opens new possibilities for high precision $\mathrm{M}$ spectroscopy.

In this Chapter we review the ongoing measurements of the $1 \mathrm{~S}-2 \mathrm{~S}$ transition and the Lamb shift (LS) of muonium in the context of the MuoniuM 1Aser SpectroScopy (Mu-MASS) experiment at PSI. A future measurement of the muonium Fine Structure (FS) is also currently under consideration. A schematic overview of these efforts is given in Figure 29.1. The Muonium Spectroscopy Experiment Using Microwave (MuSEUM) is ongoing at J-PARC [11] aiming to improve the muonium HyperFine Splitting (HFS).
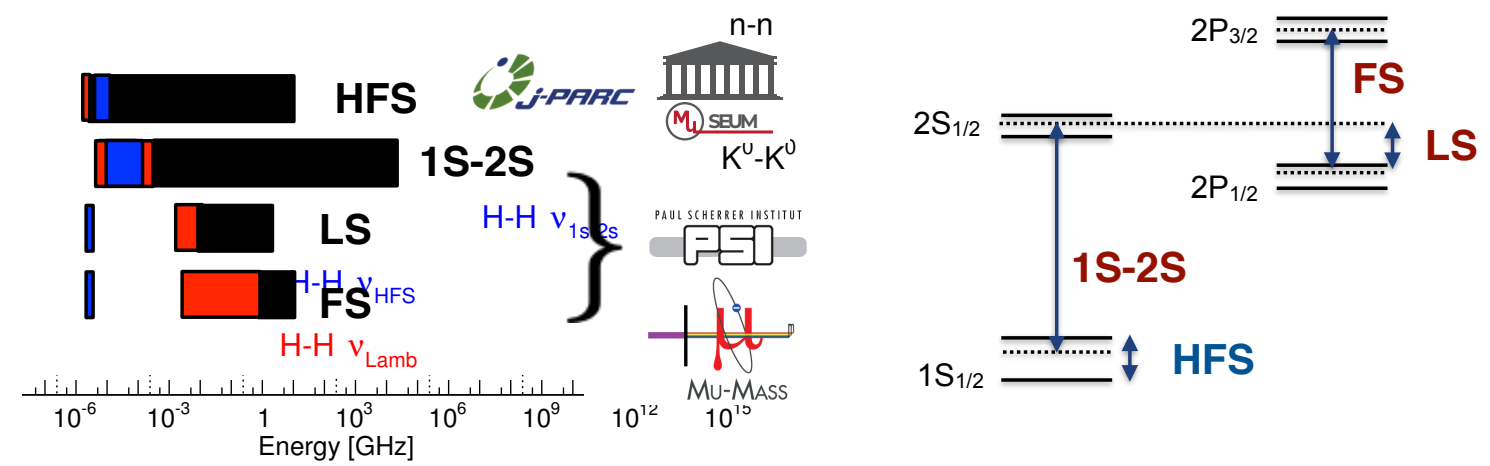

Figure 29.1: Left: M spectroscopy precision goals. Edges from right to left are: Transition frequency, highest precision literature value (black), precision goal of ongoing experiments (red), present or near-future magnitude of uncalculated QED terms (blue). Right: Schematic energy levels of $\mathrm{M}$ with planned and ongoing experiments at PSI (red) and J-PARC (blue).

\subsection{Background}

Strictly speaking, the fundamental constants that are prominent in the muonic sector at low energy are the muon mass and lifetime [12]. However, a general way of searching for physics beyond the standard model (SM) is to compare constants determined with different systems [13]. The relevant constants are the Rydberg constant $R_{\infty}$, the muon magnetic moment $\mu_{\mu}$, 
the fine structure constant $\alpha$, and the muon mass $m_{\mu}$.

Assuming the validity of high-order bound-state QED corrections, $m_{\mu}$ is [14]

$$
\frac{m_{\mu}}{m_{e}}=206.768281(2)(3),
$$

where the first uncertainty is from the experimental M ground-state hyperfine splittings measurement $\Delta v_{\mathrm{HFS}}$ [15], and the second is due to uncalculated QED terms [14], resulting in a combined relative uncertainty at a level $19 \mathrm{ppb}$. There is a strong motivation to 'free up' $\Delta v_{\mathrm{HFS}}$ from having to determine $m_{\mu}$. Currently, a $100 \mathrm{ppm}$ test of bound-state QED corrections is achieved by comparing the experimental and theoretical ground-state, zero-field $\mathrm{M}$ hyperfine splitting [14]

$$
\Delta v_{\mathrm{HFS}}^{\text {th }}-\Delta v_{\mathrm{HFS}}^{\mathrm{ex}}=96\left(51_{\mathrm{ex}}\right)\left(511_{\text {mass }}\right)\left(70_{\mathrm{QED}}\right) \mathrm{Hz} .
$$

Here, one has to use the second-best determination of $m_{\mu}$, at the level of $120 \mathrm{ppb}$, which comes from a high-field determination of the magnetic moment $\mu_{\mu}$ through the Breit-Rabi technique [15], and does not depend strongly on QED corrections. It is apparent that our lack of an independent, accurate determination of $m_{\mu}$ is limiting the ability to test QED. This is especially true considering ongoing efforts to improve both experimental and theoretical errors on the M hyperfine splitting below $10 \mathrm{~Hz}[14,16]$.

The Mu-MASS experiment is the measurement of the 1S-2S transition in $\mathrm{M}$ to the few ppt level. The reduced mass contributes to this transition at the $0.5 \%$ level and so, adopting the current value of $R_{\infty}, m_{\mu}$ may be deduced from this experiment to the level of $1 \mathrm{ppb}$. This accuracy is a 20 -fold improvement over the currently best known value given in eq. (29.1). From eq. (29.2) one can see that combining the results of Mu-MASS, MuSEUM, and the continued improvement in theoretical calculations will culminate in a $2 \mathrm{ppb}$ comparison between experiment and theory.

Assuming the validity of QED corrections, the combination of Mu-MASS and MuSEUM will determine other fundamental constants. The fine structure constant $\alpha$ can be determined to $1 \mathrm{ppb}$. Even though this is not competitive to the current best determination [17,18], it is an interesting byproduct measurement.

The current value of $R_{\infty}$ is known to 2 ppt [19], and reflects a partial resolution of the proton radius puzzle [20]. The precision goal of ongoing $M$ experiments will result in a determination of $R_{\infty}$, independent of proton structure, with a comparable accuracy of $4 \mathrm{ppt}$. Adopting this value and obtaining the proton charge radius from the $\mathrm{M}-\mathrm{H}$ isotope shift may further drive the proton radius puzzle to its resolution. The $\mathrm{M} R_{\infty}$ could also be interpreted as a ppt level test of the absolute charge equality between $e^{-}$and $\mu^{+}$, improving the previous limits by three orders of magnitudes [21]. Such a test is interesting in the context of possible lepton universality violation encountered in [22]. The $4 \sigma$ departure from unitarity in the first row of the CKM matrix may also be interpreted as a hint for lepton flavour universality violation [23].

The anomalous magnetic moment of the muon $a_{\mu}=\left(g_{\mu}-2\right) / 2$ was calculated with an accuracy of $0.37 \mathrm{ppm}$ recently in a massive effort [24]. It can be compared to the anomalous frequency $\omega_{a}$, through the relation

$$
a_{\mu}=\frac{\omega_{a} / \omega_{p}}{\mu_{\mu} / \mu_{p}-\omega_{a} / \omega_{p}},
$$

where $\omega_{p}$ is a free proton NMR frequency in the same magnetic field, and $\mu_{\mu}$ is the magnetic moment of the muon derived from the Breit-Rabi measurement [15]. The combination of the recent measurement at Fermilab [25] with the previous one at BNL [26], results in a discrepancy of $4.2 \sigma$ with the theoretical value extracted from dispersion relations [24]. However, it 
should be noted that the discrepancy is reduced if one considers the latest lattice QCD calculations [27]. This motivates further improvements to both experiments and theory. The ongoing efforts at FNAL aim for an improvement of the current determination by a factor of four. At this level, more accurate values of either $\mu_{\mu}$ or $m_{\mu}$ are needed as an input to the theory. The relationship between various quantities discussed in this section is portrayed in Figure 29.2.

In contrast to the hyperfine and gross-structure, the lamb shift in $\mathrm{M}$ is a pure bound-state QED correction, and so the desired precision to make a measurement interesting is less stringent. This is especially true for high order recoil and radiative-recoil corrections, which due to the lower mass of $\mathrm{M}$ are much larger than in $\mathrm{H}$. The theoretical value for the LS is at the 2 ppm level [2], limited by uncalculated recoil contributions, and is four orders of magnitude more precise than the experimental determination. An improvement by factor of 100 or more on the current experimental accuracy of 1\% will test QED corrections on the level of which they are currently tested by the HFS.

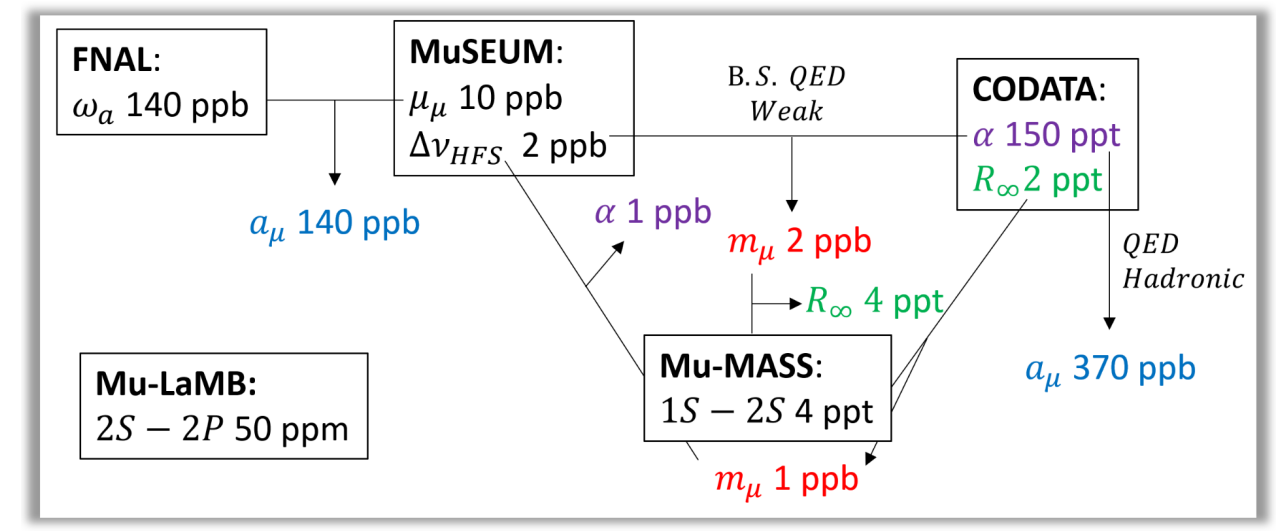

Figure 29.2: Relationship of experimental quantities measured in ongoing and planned $\mathrm{M}$ spectroscopy and storage ring experiments. Comparison of constants determined by different methods tests the validity of the theoretical calculations.

\subsection{Ongoing Mu-MASS experiment at PSI}

\subsubsection{S-2S transition}

The best experimental determination of the value of the 1S-2S line in M is 2455528941.0(9.8) $\mathrm{MHz}$ [21], in good agreement with the predictions of bound-state QED which is 2455528935.4(1.4) MHz [28,29]. The uncertainty of the theoretical value is dominated by our current knowledge of the muon mass (to $120 \mathrm{ppb)} \mathrm{extracted} \mathrm{with} \mathrm{the} \mathrm{Rabi-method} \mathrm{[15].}$ The QED calculations are known with an accuracy of $20 \mathrm{kHz}$ (8 ppt) [28-30], with prospects to improve by at least a factor of two in the near future [31].

The experiment was performed using the pulsed muon source at RAL. M atoms were formed in a $\mathrm{SiO}_{2}$ powder and emerged into vacuum with a thermal Maxwell-Boltzmann velocity distribution at 296(10) $\mathrm{K}$ and a conversion efficiency per impinging muon of $2.2 \%$. A fraction of them would then interact with a $244 \mathrm{~nm}$ counter-propagating pulsed laser beam inducing the $1 \mathrm{~S}-2 \mathrm{~S}$ transition detected via photoionization of the $2 \mathrm{~S} \mathrm{M}$ state in the same laser field. The combined excitation and detection efficiency on resonance was around $3 \times 10^{-5}$ and a total of 99 events were collected for $3 \times 10^{6}$ laser shots.

The use of a pulsed laser for such a measurement imposes several limitations [8]. The rapid optical phase changes, due to the high intensity in the pulsed optical amplifiers, result in frequency variations within the laser pulse which can reach several tens of $\mathrm{MHz}$. This so 

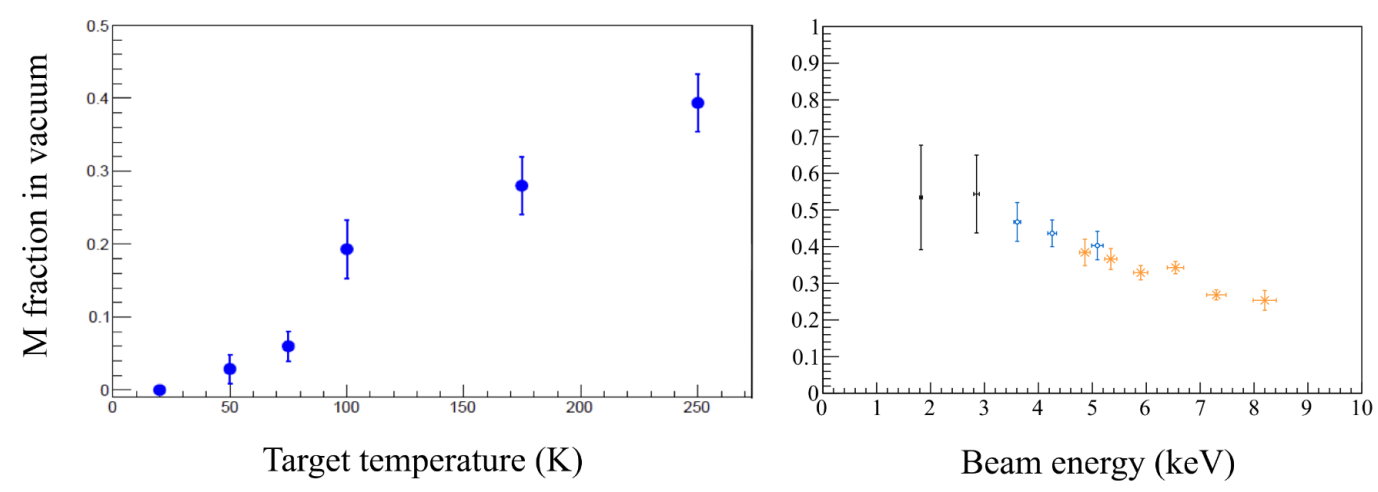

Figure 29.3: Efficient M production at the LEM beamline. Left: Fraction of muonium emitted into vacuum per incoming muon at $5 \mathrm{keV}$ from porous silica thin films as a function of temperature. Reproduced with permission from [32]. Right: Fraction of muonium emerging from a thin carbon foil as a function of the exit energy. Reproduced with permission from [33]. The fraction of $\mathrm{M}$ formed in the $2 S$ state is of the order of $10 \%$.

called chirping effect, even if measured on a pulse-by-pulse basis, introduces a systematic error at the $\mathrm{MHz}$ level. In addition, the limited interaction time of the laser pulse with the atoms and the high instantaneous power results in a broadening of the experimental linewidth to $20 \mathrm{MHz}$. These issues can be completely resolved using a continuous wave (CW) laser for which the expected linewidth will be well below $1 \mathrm{MHz}$, limited by time-of-flight broadening. A crucial step in performing CW laser spectroscopy is the development at LEM of M converters emitting 20(40)\% of the incident low-energy muons as M back into vacuum (see Figure 29.3) with a thermal Maxwell Boltzmann velocity distribution at 100(250) K and a cosine angular distribution [32,34]. The increased atom-laser interaction time will compensate for the lower available power compared to a pulsed laser. These new converters combined with the recent demonstration of high-power CW lasers at $243 / 244 \mathrm{~nm}[35,36]$ that can be cavity enhanced to more than $33 \mathrm{~W}$ of intracavity power [37] will enable an improved measurement of the 1S-2S transition frequency by three orders of magnitude which is the aim of the ongoing Mu-MASS experiment.

A schematic representation of Mu-MASS is given in Figure 29.4. A collimated beam of monoenergetic $5 \mathrm{keV} \mu^{+}$is focused using a segmented conical lens to a $6 \times 20 \mathrm{~mm}$ target coated with a thin film of mesoporous silica where $\mathrm{M}$ atoms are formed. When muons hit the target, secondary electrons are emitted and guided to a nearby Micro-channel plate (MCP) detector. These electrons give a start signal to the data acquisition system. The $\mathrm{M}$ atoms emitted into vacuum travel through the waist of a cavity-enhanced laser beam, which on resonance excites them to the $2 S$ state with an efficiency of few $10^{-6}$. A pulsed electric field is used to mix the $2 \mathrm{~S}$ and $2 \mathrm{P}$ states so that the radiative lifetime is reduced to a few ns. The Lyman-Alpha photon emitted in this quenching process is detected efficiently with a pair of CsI coated MCP detectors, giving a stop signal to the system which allows for a narrow (roughly $10 \mathrm{~ns}$ ) detection window. To suppress the background to the required level, scintillation counters surround the system to detect the emitted positron from $\mu^{+}$decay in coincidence with the electron which was bound in the M system detected in the same MCP used for the secondary electrons, following the Ly- $\alpha$ detection. The estimated event rate is of order of few per hour and allows the $1 \mathrm{MHz}$ transit-time-broadened linewidth to be resolved to below $100 \mathrm{kHz}$ within 10 days. Further improvements in the detection and laser systems would further push the uncertainty limit to the final goal of $10 \mathrm{kHz}$. 


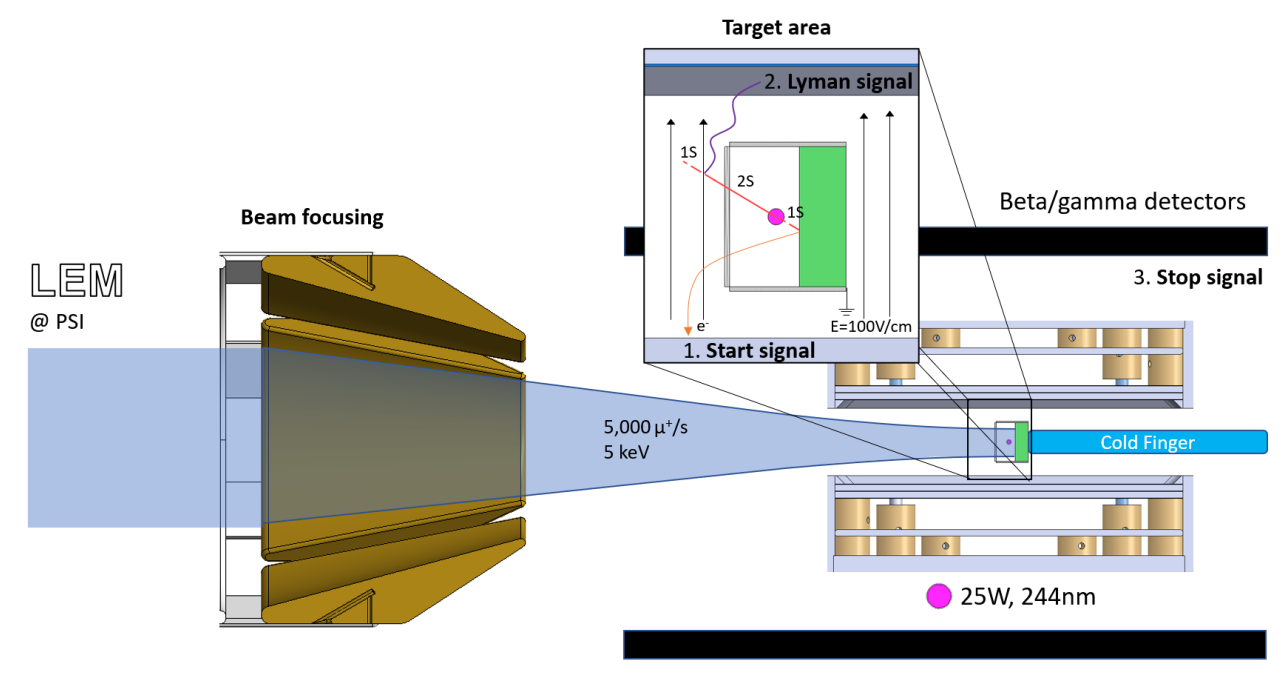

Figure 29.4: Schematic diagram of the Mu-MASS laser experiment.

\subsection{2 n=2 Lamb Shift}

The classical Lamb Shift of hydrogenic atoms $2 S_{1 / 2}-2 P_{1 / 2}$ is in the microwave (MW) range. In contrast with narrow two-photon transitions, allowed MW transitions are well-suited for measurements with a fast beam. In hydrogen, the most precise LS measurement was accomplished recently using charge exchange of a proton beam with velocity of $c / 100$ with $\mathrm{H}_{2}$ gas [38]. The linewidth of a single-pass resonance experiment is limited to $100 \mathrm{MHz}$ by the radiative lifetime of the $2 P$ state. To resolve this linewidth to a level of roughly $50 \mathrm{kHz}$, where systematic effects are expected to dominate [39], millions of $2 S-2 P$ detected transition events are needed. Clearly, M excitation from the ground state by either pulsed or CW laser is not suited for this task because of the low excitation probability.

On the other hand, it was demonstrated that $\mathrm{M}(2 \mathrm{~S})$ can be efficiently produced by using the so called beam-foil technique [40]. In this scheme, muons passing through a thin foil capture an electron to produce muonium with population in the levels with principal quantum number $n$ scaling roughly as $1 / n^{3}$. Based on hydrogen data and calculations, it is estimated that the $2 \mathrm{~S}$ fraction is roughly $5-10 \%$ [40], which agrees with experimental data [33]. After exiting the foil, $n p$ states decay rapidly, leaving a beam composed mainly of ground-state and $\mathrm{M}(2 \mathrm{~S})$ suitable for spectroscopy experiments.

Using the beam-foil technique, M in vacuum was first observed at LAMPF in 1981 with 4.0 MeV surface $\mu^{+}$at a rate of $3 \times 10^{6} / \mathrm{s}$ traveling through different foil materials [41]. A similar campaign was conducted at the same time in TRIUMF [42]. Having measured M(2S) in vacuum, both groups determined the LS, with the TRIUMF results achieving higher precision, and the value of $v_{L S}=1070_{-15}^{+12} \mathrm{MHz}$ [43], limited by statistics. Both groups used high-energy $(>2 \mathrm{MeV}) \mu^{+}$beams which had to be degraded to form $\mathrm{M}$ in the foil, creating a small signal above a large muon-related background. It is apparent that it is not only the accelerator intensity which was the limiting factor, but also the lack of a well-collimated $\mu^{+}$beam below $20 \mathrm{keV}$.

Recently at the PSI LEM beamline, it was demonstrated [33] that an intense/collimated $\mathrm{M}(2 \mathrm{~S})$ beam can be produced paving the way to an improved measurement of the LS in M which is ongoing. As shown Figure 29.5, monoenergetic $\mu^{+}$at $10 \mathrm{keV}$ traverse an ultrathin $(10 \mathrm{~nm})$ carbon foil, creating $\mathrm{M}(2 \mathrm{~S})$ atoms with $2 \%$ efficiency while emitting secondary electrons. These electrons are guided to an MCP detector by an electrostatic field too weak to significantly quench the $2 S$ beam. The $2 S$ beam then traverses a hyperfine selection transmission line which quenches $2 S_{F=1}$ states, followed by another transmission line tuned around the 
$2 S_{F=0}-2 P_{1 / 2, F=1}$ resonance around $600 \mathrm{MHz}$. On resonance the atoms reach the detection stage, which consist of a strong electrostatic quenching field and two CsI-coated MCP detectors detecting Ly- $\alpha$ photons. To reduce the background, an MCP detector in the back is used in coincidence. A Monte-Carlo simulation of the experiment predicts that the linewidth can be resolved below $1 \mathrm{MHz}$ within a few days of beamtime, constituting an improvement over the state-of-the-art by more than one order of magnitude.

(a) Neutralization \& Tagging

(b) Hyperfine-selection

(c) Scan resonance

(d) Quenching \& detection (e) Back detector

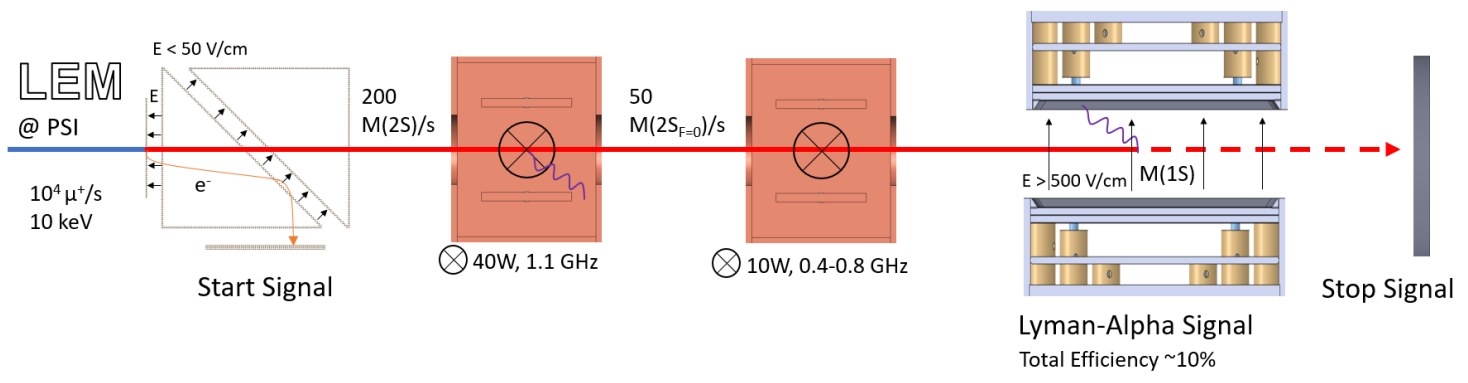

Figure 29.5: Overview of the scheme for the Lamb shift measurement.

\subsection{Summary and Outlook}

All previous $\mathrm{M}$ spectroscopic measurements were performed at pulsed muon facilities and were statistically limited. The demonstration at PSI of the production of ground state M atoms emitted into vacuum at cryogenic temperatures [32] and a high intensity metastable M $2 S$ beam [33] will allow past limitations to be overcome. The Mu-MASS final goal is to measure the $1 \mathrm{~S}-2 \mathrm{~S}$ energy to $10 \mathrm{kHz}$ which is an improvement by a factor of 1000 compared to the current results. The current projected accuracy for the Lamb shift measurement at LEM is at a level of $1 \mathrm{MHz}$. Paired with ongoing work on the HFS at J-PARC, those measurements will result in a stringent test of bound-state QED, the determination of fundamental constants, and tests of new physics.

The development of muCool [16] and the high intensity Muon Beam (HiMB) [44] will further increase the available statistics by orders of magnitude. This will help to implement more systematically robust measurement schemes, such as the employment of an enhancement cavity with a larger laser beam to reduce AC-stark shift in the 1S-2S measurement and the use of variations of Ramsey-spectroscopy to measure the Lamb shift.

\section{References}

[1] S. G. Karshenboim, Nuclear structure-dependent radiative corrections to the hydrogen hyperfine splitting, Phys. Lett. A 225, 97 (1997), doi:10.1016/S0375-9601(96)00861-4.

[2] C. Frugiuele, J. Pérez-Ríos and C. Peset, Current and future perspectives of positronium and muonium spectroscopy as dark sectors probe, Phys. Rev. D 100, 015010 (2019), doi:10.1103/PhysRevD.100.015010.

[3] A. H. Gomes, V. Alan Kostelecký and A. J. Vargas, Laboratory tests of Lorentz and CPT symmetry with muons, Phys. Rev. D 90, 076009 (2014), doi:10.1103/PhysRevD.90.076009.

[4] A. Ishida, T. Namba, S. Asai, T. Kobayashi, H. Saito, M. Yoshida, K. Tanaka and A. Yamamoto, New precision measurement of hyperfine splitting of positronium, Phys. Lett. B 734, 338 (2014), doi:10.1016/j.physletb.2014.05.083. 
[5] M. W. Heiss, G. Wichmann, A. Rubbia and P. Crivelli, The positronium hyperfine structure: Progress towards a direct measurement of the $23 S 1 \rightarrow 21 S 0$ transition in vacuum, J. Phys.: Conf. Ser. 1138, 012007 (2018), doi:10.1088/1742-6596/1138/1/012007.

[6] L. Gurung, T. J. Babij, S. D. Hogan and D. B. Cassidy, Precision microwave spectroscopy of the positronium $n=2$ fine structure, Phys. Rev. Lett. 125, 073002 (2020), doi:10.1103/PhysRevLett.125.073002.

[7] D. M. Webber et al., Measurement of the positive muon lifetime and determination of the Fermi constant to part-per-million precision, Phys. Rev. Lett. 106, 041803 (2011), doi:10.1103/PhysRevLett.106.041803.

[8] K. P. Jungmann, Precision muonium spectroscopy, J. Phys. Soc. Jpn. 85, 091004 (2016), doi:10.7566/JPSJ.85.091004.

[9] E. Morenzoni, F. Kottmann, D. Maden, B. Matthias, M. Meyberg, Th. Prokscha, Th. Wutzke and U. Zimmermann, Generation of very slow polarized positive muons, Phys. Rev. Lett. 72, 2793 (1994), doi:10.1103/PhysRevLett.72.2793.

[10] T. Prokscha, E. Morenzoni, K. Deiters, F. Foroughi, D. George, R. Kobler, A. Suter and V. Vrankovic, The new beam at PSI: A hybrid-type large acceptance channel for the generation of a high intensity surface-muon beam, Nucl. Instr. Meth. Phys. Res. Sec. A 595, 317 (2008), doi:10.1016/j.nima.2008.07.081.

[11] S. Kanda et al., New precise spectroscopy of the hyperfine structure in muonium with a high-intensity pulsed muon beam, Phys. Lett. B 815, 136154 (2021), doi:10.1016/j.physletb.2021.136154.

[12] M. Tanabashi, K. Hagiwara, K. Hikasa, K. Nakamura, Y. Sumino, F. Takahashi, J. Tanaka, K. Agashe, G. Aielli, C. Amsler, M. Antonelli and Asner, Review of particle physics, Phys. Rev. D 98, 030001 (2018), doi:10.1103/PhysRevD.98.030001.

[13] R. H. Parker, C. Yu, W. Zhong, B. Estey and H. Müller, Measurement of the fine-structure constant as a test of the Standard Model, Science 360, 191 (2018), doi:10.1126/science.aap7706.

[14] M. I. Eides, Hyperfine splitting in muonium: Accuracy of the theoretical prediction, Phys. Lett. B 795, 113 (2019), doi:10.1016/j.physletb.2019.06.011.

[15] W. Liu et al., High precision measurements of the ground state hyperfine structure interval of muonium and of the muon magnetic moment, Phys. Rev. Lett. 82, 711 (1999), doi:10.1103/PhysRevLett.82.711.

[16] A. Antognini et al., Demonstration of muon-beam transverse phase-space compression, Phys. Rev. Lett. 125, 164802 (2020), doi:10.1103/PhysRevLett.125.164802.

[17] R. H. Parker, C. Yu, W. Zhong, B. Estey and H. Müller, Measurement of the fine-structure constant as a test of the Standard Model, Science 360, 191 (2018), doi:10.1126/science.aap7706.

[18] L. Morel, Z. Yao, P. Cladé and S. Guellati-Khélifa, Determination of the finestructure constant with an accuracy of 81 parts per trillion, Nature 588, 61 (2020), doi:10.1038/s41586-020-2964-7.

[19] E. Tiesinga, P. J. Mohr, D. B. Newell and B. N. Taylor, The 2018 codata recommended values of the fundamental physical constants, Codata.org 8 (2019). 
[20] J.-P. Karr and D. Marchand, Progress on the proton-radius puzzle, Nature 575, 61 (2019), doi:10.1038/d41586-019-03364-z.

[21] V. Meyer et al., Measurement of the $1 s-2 s$ energy interval in muonium, Phys. Rev. Lett. 84, 1136 (2000), doi:10.1103/PhysRevLett.84.1136.

[22] R. Aaij et al., Measurement of CP-averaged observables in the $B^{0} \rightarrow K^{* 0} \mu^{+} \mu^{-}$decay, Phys. Rev. Lett. 125, 011802 (2020), doi:10.1103/PhysRevLett.125.011802.

[23] A. Crivellin and M. Hoferichter, $\beta$ decays as sensitive probes of lepton flavor universality, Phys. Rev. Lett. 125, 111801 (2020), doi:10.1103/PhysRevLett.125.111801.

[24] T. Aoyama et al., The anomalous magnetic moment of the muon in the Standard Model, Phys. Rep. 887, 1 (2020), doi:10.1016/j.physrep.2020.07.006.

[25] B. Abi, Measurement of the positive muon anomalous magnetic moment to $0.46 \mathrm{ppm}$, Phys. Rev. Lett. 126, 141801 (2021), doi:10.1103/PhysRevLett.126.141801.

[26] G. W. Bennett et al., Final report of the E821 muon anomalous magnetic moment measurement at BNL, Phys. Rev. D 73, 072003 (2006), doi:10.1103/PhysRevD.73.072003.

[27] Sz. Borsanyi et al., Leading hadronic contribution to the muon magnetic moment from lattice QCD, Nature 593, 51 (2021), doi:10.1038/s41586-021-03418-1.

[28] K. Pachucki, D. Leibfried, M. Weitz, A. Huber, W. König and T. W. Hänsch, Theory of the energy levels and precise two-photon spectroscopy of atomic hydrogen and deuterium, J. Phys. B: At. Mol. Opt. Phys. 29, 177 (1996), doi:10.1088/0953-4075/29/2/008.

[29] S. G. Karshenboim, The Lamb shift of excited s-levels in hydrogen and deuterium atoms, Zeitschrift für Physik D Atoms, Molecules and Clusters 39, 109 (1997), doi:10.1007/s004600050116.

[30] M. I. Eides, H. Grotch and V. A. Shelyuto, Theory of light hydrogenlike atoms, Phys. Rep. 342, 63 (2001), doi:10.1016/S0370-1573(00)00077-6.

[31] S. G. Karshenboim, Private Communication (2020).

[32] A. Antognini et al., Muonium emission into vacuum from mesoporous thin films at cryogenic temperatures, Phys. Rev. Lett. 108, 143401 (2012), doi:10.1103/PhysRevLett.108.143401.

[33] G. Janka et al., Intense beam of metastable Muonium, Eur. Phys. J. C 80, 804 (2020), doi:10.1140/epjc/s10052-020-8400-1.

[34] K. S. Khaw, A. Antognini, T. Prokscha, K. Kirch, L. Liszkay, Z. Salman and P. Crivelli, Spatial confinement of muonium atoms, Phys. Rev. A 94, 022716 (2016), doi:10.1103/PhysRevA.94.022716.

[35] Z. Burkley, A. D. Brandt, C. Rasor, S. F. Cooper and D. C. Yost, Highly coherent, watt-level deep-UV radiation via a frequency-quadrupled Yb-fiber laser system, Appl. Opt. 58, 1657 (2019), doi:10.1364/AO.58.001657.

[36] Z. Burkley, L. de Sousa Borges, B. Ohayon, A. Golovozin, J. Zhang and P. Crivelli, Stable high power deep-UV enhancement cavity in ultra high vacuum with fluoride coatings (2021), arXiv:2105.14977. 
[37] S. Cooper, Z. Burkley, A. Brandt, C. Rasor and D. Yost, Cavity-enhanced deep ultraviolet laser for two-photon cooling of atomic hydrogen, Optics Lett. 43, 1375 (2018).

[38] N. Bezginov, T. Valdez, M. Horbatsch, A. Marsman, A. C. Vutha and E. A. Hessels, A measurement of the atomic hydrogen Lamb shift and the proton charge radius, Science 365, 1007 (2019), doi:10.1126/science.aau7807.

[39] D. A. Andrews and G. Newton, Radio-frequency atomic beam measurement of the $\left(2^{2} s_{\frac{1}{2}}, f=0\right)-\left(2^{2} p_{\frac{1}{2}}, f=1\right)$ Lamb-shift interval in hydrogen, Phys. Rev. Lett. 37, 1254 (1976), doi:10.1103/PhysRevLett.37.1254.

[40] C. Fry, J. Warren, R. Kiefl, C. Oram, G. Ludgate, P. Schmor, A. Olin, G. Marshall, B. Erickson and G. Morris, Observation of epithermal 2s muonium, Hyperfine Inter. 18, 691 (1984).

[41] P. R. Bolton et al., Observation of muonium in vacuum, Phys. Rev. Lett. 47, 1441 (1981), doi:10.1103/PhysRevLett.47.1441.

[42] C. J. Oram, C. A. Fry, J. B. Warren, R. F. Kiefl and J. H. Brewer, Observation of the $2 S$ state of muonium in a vacuum, J. Phys. B: At. Mol. Phys. 14, L789 (1981), doi:10.1088/0022$3700 / 14 / 23 / 007$.

[43] C. J. Oram, J. M. Bailey, P. W. Schmor, C. A. Fry, R. F. Kiefl, J. B. Warren, G. M. Marshall and A. Olin, Measurement of the Lamb shift in muonium, Phys. Rev. Lett. 52, 910 (1984), doi:10.1103/PhysRevLett.52.910.

[44] K. Kirch, Slow muons and muonium, In Proceedings of the Seventh Meeting on CPT and Lorentz Symmetry, pp. 53, World Scientific (2017). 